\title{
Utilización de Moodle para el desarrollo y evaluación de competencias en los Alumnos
}

\author{
José M. López, Eva Romero y Eva Ropero \\ Universidad Europea de Madrid. Facultad de Ciencias Sociales. Departamento de Economía \\ de la Empresa. C/ Tajo, s/n., CP 28670 Villaviciosa de Odón, Madrid-España. \\ (e-mail: jose.lopez@uem.es, eva.romero@uem.es, eva.ropero@uem.es)
}

Recibido Jun. 06, 2010; Aceptado Jul. 06, 2010; Versión final recibida Jul. 29, 2010

\begin{abstract}
Resumen
Los autores explican en este artículo el uso de la plataforma virtual Moodle como un instrumento para el desarrollo de competencias de los alumnos. Moodle tiene muchos recursos implementados que se utilizan generalmente solo como soporte a la docencia. Los autores aportan aquí un nuevo enfoque acerca de cómo se pueden desarrollar y evaluar competencias gracias al uso de las nuevas tecnologías. Los autores incluyen sus experiencias en las diferentes asignaturas y titulaciones en las que han impartido clases, con el objetivo de desarrollar diferentes competencias tales como trabajo en equipo, responsabilidad, liderazgo, cooperación, integración de la información, habilidades comunicativas, e iniciativa, entre otras. Del estudio y análisis se concluye que Moodle contiene herramientas que han probado ser útiles para el desarrollo de competencias de los alumnos.
\end{abstract}

Palabras clave: competencias, tecnología, Moodle, enseñanza-aprendizaje.

\section{Using Moodle to develop and assess competences in students}

\begin{abstract}
The authors explain in this paper the use the Moodle platform as a tool to develop students' competences. Moodle has several options and resources that are usually employed for teaching and learning only. The authors give here a new viewpoint about using Moodle for developing and evaluate student's competences. The authors include their experiences of employing Moodle in different courses and majors in which they have lectured, in the developing student's abilities such as team working, responsibility, leadership, cooperation, communication skills, and initiative, among others. From the study and analysis it is concluded that Moodle contains tools that have proved to be good for developing some specific competences in students.
\end{abstract}

Keywords: competences, technology, Moodle, teaching-learning 


\section{INTRODUCCIÓN}

En los últimos años las nuevas tecnologías han ido desarrollándose y tomando relevancia en todos los aspectos de la sociedad, incluyendo no solo su aplicación en parcelas como la sanidad o la logística sino también en el mundo docente. La importancia y crecimiento de las nuevas tecnologías es patente como pueden mostrar los siguientes datos. Por ejemplo, según datos del Instituto Nacional de Estadística, en el año 2003 el 40\% de los hogares de la zona euro tenía conexión a Internet mientras que en el 2008 el porcentaje ascendió al 62\%. En España el aumento en el número de conexiones ha sido ligeramente superior. En el año 2003 sólo el $28 \%$ de la población tenía acceso a la red mientras que en 2008, Internet ya había llegado al 51\% de los hogares. En cuanto al número de conexiones de banda ancha se observa que en el año 2005 solo un $23 \%$ de los hogares de la zona euro y un $21 \%$ de los hogares españoles disponían de esta tecnología, mientras que en el año 2008 estas cifras aumentaron al 50\% y 45\% respectivamente.

La universidad no puede ser ajena a estos avances tecnológicos y debe adaptarlos a sus necesidades y a las de sus usuarios que cada vez demandan más este tipo de servicios. Es por esto que los autores consideran de gran importancia el análisis y la reflexión sobre cómo se debe incluir la tecnología para mejorar la gestión de recursos, la comunicación con los alumnos y el día a día en las aulas en general. Como destacan Palomo et al. (2006) "los centros educativos tienen que preparar a su alumnado para, no sólo acceder a la información, sino también saber "crear" conocimiento basado en dicha información". Cabero y Llorente (2006) también hacen referencia a cómo la universidad debe adaptarse a estos cambios cuando dicen: "Cambios en cómo los estudiantes aprenden y qué aprenden son consecuencia irrefutable de estar inmersos en una sociedad digital que está exigiendo un conjunto de destrezas y habilidades tecnológicas por parte de los alumnos hasta ahora innecesarias". Los profesores no pueden permanecer ajenos a este cambio, y así, como señala Salinas (1999) los profesores tienen que asumir su nuevo rol con el avance de las tecnologías. Algo que suele ser difícil y costoso por las reticencias que en muchas ocasiones tienen los propios profesores con el cambio en su metodología docente, tal y como señalan Cabero (2002) y Barroso (2003).

Prácticamente todas las universidades españolas han dado el paso de incluir una plataforma virtual como apoyo a la docencia. Sin embargo, en muchas de ellas no ha quedado más que como un mero repositorio de documentos, además de que un porcentaje de profesores muy alto no hace uso de la misma. Esto puede ser debido a la falta de formación de sus profesores y de herramientas organizativas y administrativas, que son claves para su incorporación a los procesos de enseñanza-aprendizaje (Dobrov, 1979; Cabero, 2004). En concreto, en los estudios de Cabero (1998 y 2001) se observa que los motivos fundamentales para el poco uso de las nuevas tecnologías por parte de los profesores en la formación de sus estudiantes son, entre otros, la falta de instalaciones adecuadas, la falta de formación, el excesivo número de estudiantes y la inexistencia de un responsable de medios tecnológicos. De la misma forma, Olsson (2007) hace notar que todavía queda mucho por hacer para que las plataformas de aprendizaje digitales sean plenamente integradas en las asignaturas, principalmente por la falta de tiempo para formación de los profesores. Pero en la Universidad Europea de Madrid, lugar donde se lleva a cabo este análisis, se resolvieron estos problemas antes de que surgieran, ya que las facultades tienen la figura de responsable tecnológico en cada facultad, las clases tienen grupos de estudiantes reducidos y las instalaciones son modernas y están suficientemente preparadas. Todo esto ha posibilitado que, en tan sólo un curso académico (2006/2007) se integrara la plataforma virtual Moodle en todas las asignaturas y fuese usada por la mayoría de nuestro personal docente (López y Ropero, 2008a). Otra posible razón de que en otras universidades no se diera un adecuado uso de las plataformas virtuales es la mala selección de la plataforma (poco intuitiva, y poco flexible).

Probablemente, Moodle sea una de las plataformas mejor diseñadas para apoyo a la docencia siguiendo el nuevo paradigma, ya que permite obtener información acerca de cómo el aprendizaje va adquiriéndose por parte de los alumnos (Dougiamas y Taylor, 2003; Zenha-Rela, y Carvalho, 2006; Mateo y Sangrà, 2009). Además, Moodle está desarrollada en código abierto y modular, lo que le otorga una gran flexibilidad para adecuarse a las distintas necesidades docentes (ZenhaRela, y Carvalho, 2006). De hecho, en la Universidad Europea de Madrid también se le da un uso en áreas de gestión, entre otras utilidades para facilitar la coordinación entre profesores (López y Ropero, 2008b). 
Por otro lado, la integración del sistema universitario español en el nuevo Espacio Europeo de Educación Superior (EEES) requiere que las nuevas titulaciones sean diseñadas de tal manera que permitan al alumno desarrollar las competencias genéricas, transversales y específicas que le posibilitarán para desempeñar su profesión tanto a nivel nacional como internacional. Acorde a lo que se entiende por competencias, según la Comisión Europea se refieren a la combinación de destrezas, conocimientos, aptitudes y actitudes, y a la inclusión de la disposición para aprender además del saber común. Una de las principales novedades de los nuevos grados es que deben estar orientados a las futuras profesiones y esto requiere que se desarrollen en el aula tanto competencias generales como competencias específicas. De este modo los alumnos recibirán una formación integral que les facilitará su ingreso en el mundo laboral.

Se pretende también que la estructura de los programas sea flexible para facilitar el reconocimiento de los créditos en distintas universidades así como el acceso a las distintas titulaciones desde diversas formaciones previas. Esto dará una mayor movilidad al sistema de educación superior dentro de la Unión Europea, consiguiendo que a nivel internacional nuestras titulaciones tengan un mayor reconocimiento. Para conseguir esto se definirán los contenidos de las titulaciones en función de las competencias científicas y profesionales que el alumno va a adquirir al cursar las diferentes asignaturas. Con todo esto, en el EEES el desarrollo de competencias en el aula se ha convertido en uno de los pilares fundamentales de la educación universitaria. Las titulaciones ya no se definen según los contenidos, sino según las competencias (generales y específicas) que los alumnos habrán adquirido al finalizar sus estudios.

En este artículo se pretende exponer cómo pueden desarrollarse las distintas competencias a través de los medios tecnológicos. A lo largo de estos tres cursos que se lleva trabajando con Moodle en nuestra Facultad, se han ido poniendo en práctica distintas actividades orientadas al desarrollo de competencias, que requerían el uso de las distintas herramientas que ofrece el campus virtual. El objetivo de este artículo es aportar un nuevo punto de vista sobre el uso de estas herramientas de Moodle ya que, aunque son extensamente utilizadas, es difícil encontrar documentación sobre cómo aplicarlas para el desarrollo de competencias. No se considerarán otras herramientas disponibles en Moodle, como los blogs y el portafolio electrónico, de las que se puede encontrar mucha información publicada (por ejemplo en Mateo y Sangrà, 2009; Soeiro, 2009).

\section{HERRAMIENTAS DE MOODLE Y LAS COMPETENCIAS}

Foro: En un foro una persona (el profesor o un alumno) abre un tema y el resto de compañeros pueden añadir comentarios sobre el mismo. Hay distintas formas de utilizar esta herramienta, cada una de ellas ayuda a desarrollar distintas competencias.

Foro de noticias: En la Universidad Europea de Madrid se denomina así al foro que el profesor utiliza para avisar a los estudiantes de noticias importantes que puedan tener que ver con la próxima clase, la actividad que están realizando, actividades universitarias, etc. Tal y como está creado, los alumnos reciben un e-mail cada vez que se incluye un nuevo mensaje en el foro. Se convierte así en una especie de tablón de anuncios en el que el profesor incluye los eventos relevantes de la asignatura. Los alumnos, además de recibir la noticia vía e-mail en el momento que el profesor decide comunicarla, pueden consultarla posteriormente siempre que lo necesiten. La utilización de este foro es más pasiva por parte de los estudiantes, por lo que es el que menos competencias permite desarrollar. Sin embargo, sí que desarrolla un sentido de la responsabilidad, al tener que mirar el e-mail con cierta regularidad. Esto es sobre todo importante en el primer curso universitario.

Foro de dudas: Muchos profesores abren un foro para que los alumnos puedan compartir sus dudas. Los alumnos pueden de esta forma resolver las dudas de sus compañeros. El alumno que plantea su duda, está desarrollando la iniciativa y el sentimiento de responsabilidad. El alumno que resuelve la duda, también está desarrollando la iniciativa, y las competencias de cooperación, liderazgo y habilidades comunicativas, ya que resulta normalmente más complicado explicarse por escrito que de forma oral. 
Foro de trabajo en grupo: Otra posibilidad que da esta herramienta es habilitar un foro para el trabajo en grupo. De esta forma, los alumnos pueden continuar con la realización del trabajo en equipo incluso en momentos en los que no están físicamente reunidos. Esta actividad fomenta el trabajo en equipo, la cooperación y las habilidades comunicativas.

Foro de debate: En numerosas asignaturas resulta de especial interés debatir ciertos temas. Sin embargo en ocasiones la falta de tiempo imposibilita la realización de estos debates en el aula. Los foros de Moodle presentan una nueva forma de debatir distintos temas, ya que los debates no son en tiempo real. Esto hace que los alumnos puedan consultar lo que han escrito sus compañeros, interiorizarlo y responder en distintos momentos del tiempo. Desarrollan así competencias generales como son la comprensión escrita, las habilidades comunicativas y la iniciativa, así como competencias específicas como la comprensión del tema en debate. En este tipo de foros se pueden debatir temas propuestos por el profesor o bien temas propuestos por los alumnos, que en muchas ocasiones les resultan más interesantes y les enriquecen más.

Wiki: Es una herramienta tecnológica que permite crear un único documento entre varios participantes. Resulta de especial utilidad para el desarrollo de contenidos de distinta naturaleza entre los alumnos de toda la clase, o entre grupos; ya que el profesor puede conocer en todo momento qué ha escrito cada alumno y evaluarlo según su participación.

Con los wikis se mejoran tanto las habilidades comunicativas, como el razonamiento crítico, ya que el desarrollo de un tema en grupo de forma colaborativa no solo implica escribir algo nuevo, sino que requiere mejorar lo que ya han escrito el resto de compañeros. Cada componente del grupo puede añadir contenidos pero también puede eliminar contenidos de sus compañeros que no considere relevantes. Se promueve así el trabajo colaborativo, ya que el resultado final no depende del trabajo individual, sino del trabajo de todo el grupo. Más información acerca de este tema se puede encontrar en De la Cruz y otros, (2007 y 2008), que tratan del modo en el que esta herramienta permite desarrollar el aprendizaje de modo colaborativo comparándola con otras herramientas como los foros y los blogs.

Chat: Como es de conocimiento común, el Chat es una herramienta que permite la comunicación simultánea a través de Internet entre dos o más personas. Moodle pone a nuestra disposición esta herramienta para su uso en el entorno académico. Esta herramienta resulta especialmente interesante en asignaturas no presenciales ya que permite un acercamiento entre el profesor y los alumnos al permitirles comunicarse en tiempo real. Son múltiples las aplicaciones que puede tener el Chat en el ámbito de la enseñanza universitaria. Se enumeran aquí algunas de las que se han empleado y las competencias que han permitido desarrollar con los alumnos.

Chat de dudas: Este tipo de chat permite al alumno consultar sus dudas en tiempo real. El profesor debe comprometerse a conectarse de forma periódica en momentos del tiempo determinados, para que los alumnos puedan consultar sus dudas. Puede resultar también interesante que sean los alumnos los que se comprometan en turnos rotativos a conectarse al Chat para resolver las dudas de sus compañeros, en caso de que sepan hacerlo y gestionarlas con el profesor en caso de que no sepan. No obstante es recomendable que el profesor esté conectado en todas las sesiones para intervenir si es necesario. En esta situación el alumno desarrolla competencias generales como la comunicación escrita, la comprensión escrita, la iniciativa y la responsabilidad, así como competencias específicas derivadas de la resolución de las dudas propuestas. Es recomendable que las distintas sesiones del Chat de dudas permanezcan visibles a los alumnos para que puedan consultarlas en cualquier momento.

Chat de debate: El Chat resulta una herramienta ideal para realizar debates online en tiempo real, ya que permite a varias personas estar conectadas a la vez. Esto posibilita el desarrollo de un debate prácticamente como si los participantes estuvieran en la misma sala, para lo que deben estar conectados en el mismo momento. Es importante en esta actividad estipular unos turnos de palabra, como si de un debate presencial se tratase, para que todos los alumnos puedan participar, pero siempre que hayan leído y comprendido las aportaciones de sus compañeros. Se 
desarrollan aquí competencias similares a las desarrolladas en los foros de debate como son la comprensión escrita, las habilidades comunicativas y la iniciativa, además de la comprensión e interiorización del tema en debate. Sin embargo, es un complemento perfecto al foro de debate, ya que en el chat no hay tanto tiempo para la reflexión. Por tanto, gracias a esta herramienta los alumnos comprenderán lo importante que es el desarrollo de la capacidad de comunicación escrita para hacerse entender rápidamente. Los temas a debate también aquí pueden ser propuestos por el profesor o por los estudiantes e incluso pueden ser moderados por uno u otros. $\mathrm{Si}$ el debate es moderado por los estudiantes se desarrolla además la competencia de la responsabilidad. Como se puede comprobar estas utilidades son muy similares a las comentadas previamente para los foros, ya que ambas son herramientas que nos permiten la comunicación con los alumnos con la única diferencia de la temporalidad y la instantaneidad.

Cuestionario: Los cuestionarios son una herramienta especialmente útil para los profesores ya que una vez diseñados permiten realizar una evaluación automática de los alumnos. Podemos enfocar los cuestionarios de dos formas:

Cuestionarios de entrenamiento: Estos cuestionarios podrán ser resueltos por el alumno tantas veces como él considere necesario. La calificación obtenida con este tipo de cuestionarios servirá tan sólo para orientar al alumno de cuál es su conocimiento de la asignatura. Le ayudarán por tanto a desarrollar competencias generales como la responsabilidad y la planificación pero sobre todo le ayudarán a desarrollar de forma autónoma las competencias específicas que le posibilitarán la superación con éxito de la asignatura. En los cuestionarios de entrenamiento es recomendable que se permita al alumno realizar cada intento sobre los anteriores, permitiéndole así estudiar cuál ha sido su respuesta en el intento previo y tomar una decisión acerca de si debe cambiarla o no. Es también recomendable que entre intento e intento se permita al alumno conocer su calificación y se le dé alguna orientación sobre la respuesta seleccionada previamente.

Cuestionarios de evaluación: Se recomienda que en este tipo de cuestionarios sólo se permita al alumno emplear un intento y con un tiempo limitado. Este tipo de cuestionarios están más orientados a la evaluación que al desarrollo de competencias. No obstante al estar normalmente fijados para unas fechas concretas desarrollan como cualquier prueba de evaluación la responsabilidad de los estudiantes. Los cuestionarios permiten poder dar rápidamente feedback a los alumnos, además de ser para ellos oportunidades de aprendizaje. Les marcan una fecha para la que tienen que haber asimilado unos contenidos, y pueden ver en el mismo momento en que terminan el cuestionario qué tal lo han entendido todo. El profesor además puede adjuntar comentarios junto con las respuestas adecuadas a cada pregunta. También se pueden utilizar los cuestionarios para realizar evaluación entre iguales, lo que permite construir conocimiento de forma conjunta. Una discusión sobre cómo hacerlo se encuentra en Zenha-Rela y Carvalho (2006).

Glosario: Esta herramienta permite que los alumnos vayan construyendo un glosario común de terminología relacionada con la materia. Este glosario permanecerá a su disposición a lo largo de la asignatura permitiendo que en cualquier momento puedan consultar los conceptos que necesiten. Durante la construcción del mismo, los alumnos aprenden a buscar información, extraer la que resulta relevante, comprenderla e integrarla, desarrollando así la competencia de integración de la información. Posteriormente deben ser capaces de redactar de forma clara, estructurada y original la información encontrada.

De este modo deberán comprender el concepto en cuestión en profundidad para ser capaces de explicarlo con claridad y originalidad, desarrollando así la consecuente competencia especifica. Además deben ser consecuentes con el trabajo realizado ya que el glosario, aún siendo supervisado por el profesor, lo construyen íntegramente los alumnos y sus compañeros lo usarán posteriormente para estudiar los distintos conceptos. De este modo cada alumno debe estar seguro de que lo incluido en el glosario es correcto y además está claramente definido para que sus compañeros no tengan dudas cuando lo consulten. Se desarrolla aquí por tanto la responsabilidad. 
También se puede trabajar en el glosario formando grupos pequeños que se encarguen de incluir los términos de forma conjunta, desarrollando así la competencia de trabajo en equipo. Esta competencia se desarrollará en cualquier caso, ya que, como el resto de los compañeros tendrán el glosario como referencia de consulta deben exigir a sus compañeros que el trabajo realizado tenga la calidad suficiente para poder ser consultado de forma adecuada. Deben por tanto aprender a ser críticos con el trabajo propio y con el de los demás.

Consultas: Moodle también permite realizar consultas a los alumnos. En estas consultas el profesor plantea una cuestión que sea de interés general y los alumnos pueden elegir la respuesta que mejor se adapte a sus necesidades por medio de una votación.

Algunas consultas que pueden resultar de interés en todas las asignaturas son:

1.- ¿Le parece adecuado el ritmo que lleva la asignatura?

2.- ¿Prefiere más teoría, más práctica o le parece adecuada la proporción entre ambas que tiene la asignatura?

3.- Seleccione la fecha que desee para la realización de la siguiente práctica o prueba de conocimiento.

En todas ellas las posibles respuestas deben ser cerradas. De esta manera, se pueden realizar votaciones sobre el funcionamiento de la asignatura. Esto hará que los alumnos se impliquen más en el desarrollo de las clases, al entender que su opinión es tenida en cuenta por el profesor, de cara a modificar en cada caso lo que se considere oportuno. Esto facilita enormemente la comunicación entre profesor y alumnos, sobre todo en aquellos casos en los que los alumnos o bien por timidez o por otros motivos no se atreven a hacer saber sus inquietudes al profesor. Con las consultas se desarrolla la responsabilidad de los alumnos, pues si sus opiniones son tenidas en cuenta deben meditarlas cuidadosamente antes de ser puestas en común. Además se fomenta la iniciativa, ya que al ver los alumnos que sus opiniones son escuchadas tomarán la iniciativa de comentar con el profesor otras sugerencias que pueden resultar de gran interés para el seguimiento y enriquecimiento de la asignatura.

Documentación: Si hacemos una reflexión rápida sobre la utilidad de los campus virtuales en el ámbito de la docencia la primera idea es su utilización para compartir documentación con los alumnos. No obstante, después de lo ya enumerado se puede comprobar que aportan muchas otras funcionalidades. En cualquier caso sí se ha de tener presente que esta es una de las principales utilidades y probablemente la más extendida en uso.

El profesor siempre debe tener una planificación de cómo será el desarrollo de la asignatura desde el principio, pero Moodle le hará estructurarla de una forma más visible para el alumno. Esto hace que este aprenda a planificarse mejor, ya que conoce la estructura de la asignatura casi desde su comienzo, teniendo una idea clara de lo que va a aprender y en qué momento. Además hace que los estudiantes tengan la responsabilidad de anticiparse a las explicaciones del profesor en los casos que resulte adecuado para una mayor comprensión de los distintos temas.

Agenda: En el campus virtual hay un calendario donde los profesores pueden poner las fechas de las actividades más importantes de la asignatura. También incorpora de forma automática los eventos que considera relevantes como la apertura y cierre de cuestionarios, y tareas, además de avisar de las actividades que se celebran en la universidad, dando así a alumnos y profesores una visión de global que les facilita la gestión de su tiempo. Esta agenda desarrolla la competencia de responsabilidad, al permitir a los alumnos que puedan organizar su tiempo entre las distintas asignaturas, y actividades que tienen que realizar. Asimismo, les permite planificarse y organizar su tiempo de la manera que consideren más adecuada, ya que les proporciona una visión general de las actividades más importantes que tendrán lugar, no sólo en la asignatura sino también en la universidad. 


\section{CONCLUSIONES}

Del análisis realizado acerca de cómo desarrollar competencias con las distintas herramientas de la plataforma virtual Moodle, se pueden extraer las siguientes conclusiones:

1) La plataforma virtual Moodle incluye herramientas útiles para el desarrollo de competencias de los alumnos.

2) El uso de estas herramientas enriquece las asignaturas tanto dentro como fuera del aula, permitiendo al alumno el desarrollo de competencias específicas y generales.

3) Los foros, chat y consultas mejoran la comunicación de los alumnos entre sí y la de estos con el profesor. Con estas herramientas se desarrollan sobre todo competencias como la comunicación oral y escrita, la responsabilidad y la iniciativa.

4) Los Wikis y Glosarios posibilitan a los alumnos el trabajo en equipo y colaborativo, haciendo que su trabajo dependa del de los demás y viceversa.

5) Empleando los documentos, los foros de noticias y la agenda el alumno desarrolla la planificación, ya que la combinación de estos tres elementos le mantiene completamente informado de todo lo que sucede en la universidad.

6) Los cuestionarios de entrenamiento permiten al alumno realizar una autoevaluación tantas veces como considere necesario y obtener de forma rápida un informe de cómo está asimilando la asignatura. Se desarrolla así la responsabilidad, la planificación y el aprendizaje autónomo.

7) Todas las herramientas mejoran la competencia que supone el manejo de las nuevas tecnologías.

Las futuras líneas de investigación que se plantean los autores pasan por obtener feedback sobre qué ha supuesto la utilización de esta herramienta en la mejora del desarrollo de las competencias. Para ello, planean realizar un posterior trabajo en el que se usarán las encuestas de calidad de los estudiantes para valorar el grado de satisfacción de los alumnos y la efectividad de esta herramienta como medio de desarrollo de las competencias, tanto genéricas como específicas

\section{REFERENCIAS}

Barroso, J., Las Nuevas Tecnologías de la Información y la Comunicación y la Formación del Profesorado Universitario. Actas del III Congreso Internacional Virtual de Educación. Internet, 1-11 Abril (2003).

Cabero, J., M. J. Gallego y F. Pavón, Los usos de los medios audiovisuales, informáticos y las nuevas tecnologías en los centros andaluces. Sevilla Kronos (2000).

Cabero, J., J.O. Barroso y P.G. Román, Las influencias de las nuevas tecnologías en los entornos de formación. Posibilidades, desafíos, retos y preocupaciones. Comunicación y Pedagogía. Nuevas Tecnologías y Recursos didácticos: 175, 48-54 (2001).

Cabero, J., Diseño y evaluación de un material multimedia y telemático para la formación y perfeccionamiento del profesorado universitario para la utilización de las nuevas tecnologías aplicadas a la docencia. Kronos, Sevilla, España (2002).

Cabero, J., Cambios organizativos y administrativos para incorporación de las TICs a la formación. Medidas a adoptar. Edutec. Revista Electrónica de Tecnología Educativa ISSN: 1135-9250 (En línea), 18 (2004). http://edutec.rediris.es/Revelec2/numeros_anteriores.html. 
Cabero, J. y M.C. Llorente, La rosa de los vientos. Dominios tecnológicos de las TIC por los estudiantes. 1-152, GID Universidad de Sevilla, Sevilla, España (2006).

De la Cruz, O., L. Gava, M. P. López y E. Ropero, Experiencias de trabajo colaborativo con Moodle en Economía, Actas de las Jornadas de Innovación Universitaria, Universidad Europea de Madrid, Madrid, España, 18-19 junio (2007).

De la Cruz, O.; L. Gava, M.P. López y E. Ropero, Wiki: Una Herramienta Colaborativa de Construcción de Conocimiento en la Universidad, Experiencias de Innovación Docente en la Universidad de Alcalá, Editorial Universidad de Alcalá, Alcalá de Henares, España. 409-422 (2008).

Dobrov, G.M, La technologie en tant qu'organisation. Revue Internationale des Sciences Sociales, XXXI: 4, 628-648 (1979).

Dougiamas, M y P. Taylor, Moodle: Using Learning Communities to Create and Open Source Course Management System. World conference on educational multimedia, hypermedia and telecommunications, Vol. 1, 171-178, (2003).

López, J. y E. Ropero, Innovación tecnológica en la docencia: una experiencia positiva en la Facultad de Economía, Derecho y Empresariales. Actas de las $\mathrm{V}$ Jornadas Internacionales de Innovación Universitaria, Universidad Europea de Madrid, España, Septiembre (2008a).

López, J. y E. Ropero, Experiencias con Moodle: Un lugar de encuentro para profesores de una misma facultad. Actas de las $\vee$ Jornadas Internacionales de Innovación Universitaria, Universidad Europea de Madrid, España, Septiembre (2008b).

Mateo, J. y A. Sangrá, Designing online learning assessment through alternative approaches: facing de concerns. European Journal of Open, Distance and e-Learning (EURODL), 3-14 Diciembre (2007).

Olsson, L. The Learning Resource Centre as a strategic tool to implement and change the use of ICT in Teacher Education, Proceedings of Society for Information Technology \& Teacher Education International Conference, 2596-2602 (2007).

Palomo, R.; R. Ruiz y J. Sánchez, Las TIC como agentes de innovación educativa. 1-219, Dirección General de Innovación Educativa y Formación del Profesorado, Sevilla, España (2006).

Salinas, J., Rol del profesorado universitario ante los cambios de la era digital. Perfeccionamiento Integral del Profesor Universitario, Primer Encuentro Iberoamericano. Universidad Central de Venezuela. Caracas, 10-18 Julio (1999).

Soeiro, A., Using New Assessment Methods in Engineering. 2nd International Multi-Conference on Engineering and Technological Innovation: IMETI, Florida (2009).

Zenha-Rela, M y R. Carvalho, Work in Progress: Self Evaluation through Monitored Peer Review Using the Moodle Platform. Frontiers in Education Conference, 36th Annual, San Diego, California, 28-31 Octubre (2006). 